\title{
A TRADUÇÃO DA CULTURA HAUDENOSAUNEE PELA LITERATURA CONTEMPORÂNEA DE ERIC GANSWORTH
}

\section{Janice Cristine Thiél}

Pontifícia Universidade Católica do Paraná (PUCPR), Brasil

janthiel09@gmail.com

DOI: https://doi.org/10.26512/caleidoscopio.v2i1

RESUM0: A literatura indígena expressa visões de mundo construídas por elementos multimodais, segundo tradições próprias. Os 'wampum belts', utilizados pela diplomacia indígena, revelam linguagem dramática e poética e merecem ser lidos sob uma perspectiva histórica e cultural, além de poderem ser interpretados como uma das primeiras formas de construção literária norte-americana. Este artigo propõe uma leitura da construção discursiva da obra Nickel eclipse: Iroquois moon (2000), de Eric Gansworth, em seu diálogo com os 'wampum belts' e outros símbolos da cultura Haudenosaunee. Pretende-se contribuir para a percepção da construção poética multimodal da literatura indígena pela análise da obra de Gansworth em sua interface com a mitologia Haudenosaunee.

Palavras-chave: Tradução, literatura indígena, Haudenosaunee, wampum belts, Gansworth.

\section{HAUDENOSAUNEE CULTURE AND ITS LITERARY INTERPRETATION BY ERIC GANSWORTH}

ABSTRACT: Literature written by the indigenous peoples of North America expresses worldviews constructed by multimodalities, according to their own traditions. Wampum belts express the language of native diplomacy and reveal dramatic and poetic elements as well. Besides, the wampum belts might also be interpreted as one of the first forms of North American literary expression. Therefore, the aim of this article is to provide an analysis of the discursive construction of Nickel Eclipse: Iroquois Moon, by Eric Gansworth (2000), in order to establish a dialogue with the tradition of the wampum belts and with the Haudenosaunee culture. In this way, we hope to contribute to the perception of the multimodal poetic language of Native American literature and its interface with Haudenosaunee mythology.

Keywords: Translation, Native American literature, Haudenosaunee, wampum belts, Gansworth. 


\section{Introdução}

Nações indígenas das Américas têm expresso suas visões de mundo e se manifestado artisticamente há séculos. Suas representações culturais podem ter sido consideradas 'primitivas' segundo conceitos eurocêntricos de história, escritura e literatura. Contudo, como afirma Antonio RISÉRIO (1993, p. 34), “Me agrada, em especial, quando, diante da estreiteza de boa parte da erudição ocidental, Rothenberg proclama: 'PRIMITIVE MEANS COMPLEX' ".

Pela tradição oral, narrativas, mitologias, representações do mundo e das relações humanas têm sido construídas por comunidades nativas que transmitem seus conhecimentos e percepções de geração a geração. Indivíduos também assumiram seu papel de contadores de histórias, narradores, poetas e dramaturgos em obras formadas por tradições ancestrais e pelo contato intercultural com nações colonizadoras. Tais autores traduzem as mitologias nativas para leitores de suas etnias e de outras culturas e utilizam estratégias discursivas e recursos poéticos híbridos, de acordo com estilos culturais e individuais, contextos de produção e recepção de suas obras.

A proposta deste artigo é abordar a construção discursiva da obra Nickel eclipse: Iroquois moon (2000), de Eric Gansworth, em seu diálogo com os 'wampum belts' da cultura Haudenosaunee, obra que pode ser vista como tradução intersemiótica da história e simbologia indígena. Pretendemos contribuir para a percepção de como a literatura indígena pode ser lida em sua construção poética multimodal pela discussão de elementos gráficos e pictográficos da obra de Gansworth que traduzem para a contemporaneidade a mitologia Haudenosaunee. Consideramos relevante observar a construção poética da obra e a relação entre povos de tradições culturalmente distintas. Como referencial teórico, buscamos fundamentação na área de tradução na obra Tradução intersemiótica (2003), de Julio Plaza, e sobre a cultura Haudenosaunee na obra Wampum belts of the Iroquois (1999) de Tehanetorens (Ray Fadden), autor indígena da tradição Mohawk, entre outras obras.

Desde o século XVIII há registros de produção textual nativa norteamericana, utilizando modalidades discursivas ocidentais e a língua inglesa. 
Segundo PERKINS et al. (2002, p. 724), o primeiro autor indígena norte-americano a publicar um texto em inglês foi Samson Occom, de etnia Mohegan. A obra $A$ sermon preached at the execution of Moses Paul, an Indian é de 1772. Uma das primeiras mulheres a publicar foi a escritora Jane Johnston Schoolcraft, ou Bamewa-wa-ge-zhik-a-quay, que significa Woman of the Stars Rushing Through the Sky (Mulher das Estrelas que Percorrem o Céu), da etnia Ojibwe. Inúmeros textos autobiográficos, de ficção e poesia são publicados a partir do século XIX. Em 1833, William Apess publica ensaios de protesto e resistência. Em 1854, John Rollin Ridge, autor de textos de ficção e poesia, publica o primeiro romance em língua inglesa escrito por um nativo, intitulado The life and adventures of Joaquin Murieta. Esses e outros autores contribuem com suas obras para a construção de uma linhagem de escritores indígenas norte-americanos que escrevem obras que dialogam com as tradições literárias nativas e europeias.

No início do século XX, aumenta a produção de romances escritos por autores indígenas: John Joseph Matthews, de etnia Osage, e D’arcy McNickle, de etnia Cree-Salish, produzem romances voltados para a temática da busca de índios miscigenados por um lugar na sociedade; Todd Downing, de etnia Choctaw, escreve romances policiais e de mistério; e Will Rogers, da nação Cherokee, publica várias sátiras relativas à política nacional e internacional.

A partir dos anos 1960, o movimento político das nações indígenas norteamericanas ganha visibilidade impulsionado por movimentos pelos direitos civis, especialmente de grupos afro-americanos e feministas. Dois autores destacam-se: Vine Deloria Jr. e N. Scott Momaday. O primeiro publica em 1969 o texto Custer died for your sins: an Indian manifesto, que assinala a condição na qual os índios se encontram na época, combate os estereótipos que constroem a identidade nativa e questiona a colonização e seus efeitos sobre as nações nativas. Por sua vez, Momaday recebe o prêmio Pulitzer de literatura em 1969 pela obra The House made of dawn, ilustrada pelo autor, que narra sua história pessoal, histórias de comunidades indígenas e de suas relações com o poder hegemônico ocidental e expressa perspectivas de contatos interculturais.

Contudo, essa linha cronológica de produção literária indígena é contada a partir do contato com as tradições europeias. E quanto ao marco zero dessa caleidoscópio: linguagem e tradução / v. 2 | n. 1 [jan. - jun. 2018] p. 35-49 | ISSN: 2526-933X 
literatura e da chamada literatura norte-americana? N. Scott MOMADAY questiona tal marco construído por referências eurocêntricas e afirma em um discurso de resistência à sujeição canônica ocidental que "A literatura norte-americana começa com a primeira percepção da paisagem americana expressa e preservada pela linguagem"1 (1997, p.14, tradução nossa). Essa afirmação leva-nos a considerar que há inúmeras formas de comunicação e a tradição oral constitui o alicerce da literatura nativa. Narrativas passadas de geração a geração têm um dinamismo próprio e significam a sustentabilidade cultural, a sobrevivência da história e da cultura das comunidades e da relação entre o narrador e o ouvinte/leitor. Contudo, a linguagem da oralidade não é a única fundadora da expressão nativa.

As culturas indígenas norte-americanas têm narrado e lavrado sua história por meio de recursos discursivos pertencentes a poéticas diversas da poética europeia, mas não menos complexas. As poéticas extraocidentais são geralmente chamadas etnopoéticas; estão à margem da poética europeia de ideologia dominante. Porém, todas as poéticas podem ser consideradas etnopoéticas, configuradas de acordo com normas próprias a cada cultura.

A heterogeneidade da produção artística e literária nativa norte-americana manifesta-se pela interação de multimodalidades, por imagens, desenhos geométricos, elementos sonoros, musicais e performáticos. Suportes geralmente considerados não literários pela tradição canônica europeia podem ter sido e ainda são utilizados para comunicação cotidiana, para construção de tratados diplomáticos, escrituras sagradas e expressão estético-literária de etnias nativas das Américas.

A pluralidade cultural e linguística nativa manifesta-se também pela multiplicidade e complexidade de gêneros textuais indígenas que quebram as fronteiras entre história, antropologia, ciência e literatura. Nesse sentido, a expressão literária indígena norte-americana pode ter sido inicialmente expressa por um suporte não relacionado pela tradição eurocêntrica à literatura: o 'wampum belt'.

\footnotetext{
${ }^{1}$ American literature begins with the first human perception of the American landscape expressed and preserved in language." 


\section{A tradução intersemiótica da mitologia Haudenosaunee}

Estima-se que as nações indígenas da América do Norte pré-contato com os europeus ocupavam 10 regiões, com uma população provavelmente entre 10 e 18 milhões de habitantes. Segundo Eric C. HENSON et al. (2008, p. 6), o censo de 2000 indicou uma população de cerca de 4.1 milhões de nativos norte-americanos e em 2005 havia 561 tribos e comunidades indígenas reconhecidas pela federação nos EUA, comunicando-se em 300 línguas, de 8 famílias linguísticas. Portanto, confirma-se a pluralidade de culturas nativas nas Américas e nos Estados Unidos na contemporaneidade.

Os Haudenosaunee, da região nordeste dos Estados Unidos e sudeste do Canadá, são muitas vezes conhecidos como Iroquois, porém esta denominação era utilizada pelos seus inimigos, os Huron, e Iroquo significava "cascavel", como forma de insulto (Cf. Charles River Editors, 2017). Há ainda a autodenominação 'People of the Longhouse' (Pessoas da Casa Comprida), que faz referência ao formato de suas casas.

Os membros da Confederação Haudenosaunee estão unidos sob 'The Great Law of Peace' (A Grande Lei da Paz), a constituição da Confederação das Seis Nações Iroquois: as nações Mohawk, Oneida, Onondaga, Cayuga, Seneca e Tuscarora. Os Haudenosaune demonstraram ao longo dos séculos sua resiliência e preocupação com a sustentabilidade cultural e sempre tiveram uma posição muito clara sobre sua soberania. As nações dessa confederação e as demais nações indígenas localizadas no que chamamos hoje Estados Unidos da América e Canadá tinham suas próprias concepções de governança, soberania e práticas diplomáticas internacionais muito antes do contato com culturas europeias. Com relação à soberania, pela visão indígena, suas leis foram dadas pelo Criador para serem vividas. Quanto às formas de governo indígena, existiam desde arranjos informais até sistemas complexos. Conselhos, federações constitucionais e democracias parlamentaristas podem ser encontradas hoje, como indicam HENSON et al. (2008, p. 18).

0 primeiro tratado ratificado entre as recém emancipadas treze colônias norte-americanas e uma tribo indígena aconteceu em 1778. Desde então, inúmeros 
tratados foram escritos, negociados e ratificados entre nações consideradas soberanas. A elaboração, os protocolos e cerimônias, bem como os símbolos e discursos que cercam os tratados entre os Estados Unidos e as nações indígenas são sustentados em ambas as partes por valores e princípios.

Da perspectiva indígena, a aliança formada pelo tratado não indicava uma aliança temporária, mas implicava uma dinâmica de parentesco entre as nações apontando para a continuidade nas relações estabelecidas. Para os nativos, a terra não era uma commodity, nem era negociável. Em geral, há uma relação afetiva dos nativos com a terra, considerada sagrada, mas seu vínculo é também político, pois envolve soberania, governança e autoridade.

Segundo a tradição europeia, ocidental, como gênero textual, o tratado constitui documento histórico primário, texto legal ou diplomático. 0 tratado é firmado por escrito e assinado por autoridades ou recebe o selo que garante sua autenticidade; o documento é arquivado para que possa ser utilizado, ou para servir como referência após sua validação. Já, para as nações indígenas, os textos dos tratados são registrados pela oralidade, pela memória e por registros materiais diversos, tais como aqueles encontrados em 'wampum belts'. 0 termo 'wampum', cunhado pelo idioma Narragansett, refere-se a contas feitas com conchas por índios da região do nordeste dos Estados Unidos, contas brancas e púrpuro-negras. O 'wampum' foi inicialmente utilizado como enfeite e para trocas comerciais. Segundo TEHANETORENS (1999, p. 11), colonos europeus fabricavam 'wampum' e utilizavam-no como a primeira moeda. Contudo, para os Iroquois/Haudenosaunee, era utilizado em cerimônias oficiais e religiosas. 0 'wampum' de cor branca era utilizado para simbolizar pureza e paz, enquanto o 'wampum' púrpura era utilizado em cerimônias de natureza cívica.

No que concerne especificamente aos tratados firmados com os Haudenosaunee, 'Kayahnerenhkowah' (A Grande Lei da Paz) constitui um tratado multilateral entre as nações da Confederação e tem sido transmitido através das gerações pela tradição oral. A bandeira da Confederação Haudenosaunee, baseada no 'Hiawatha wampum belt', representa as cinco nações originais que foram unidas pelo pacificador, Hiawatha. A árvore no centro representa a nação Onondaga, onde o pacificador plantou a Árvore da Paz, sob a qual os líderes das 
Cinco Nações (a nação Tuscarora foi incorporada mais tarde) enterraram suas armas de guerra. Os quatro retângulos representam as demais nações e todas estão conectadas por caminhos que se estendem para fora, para o outro.

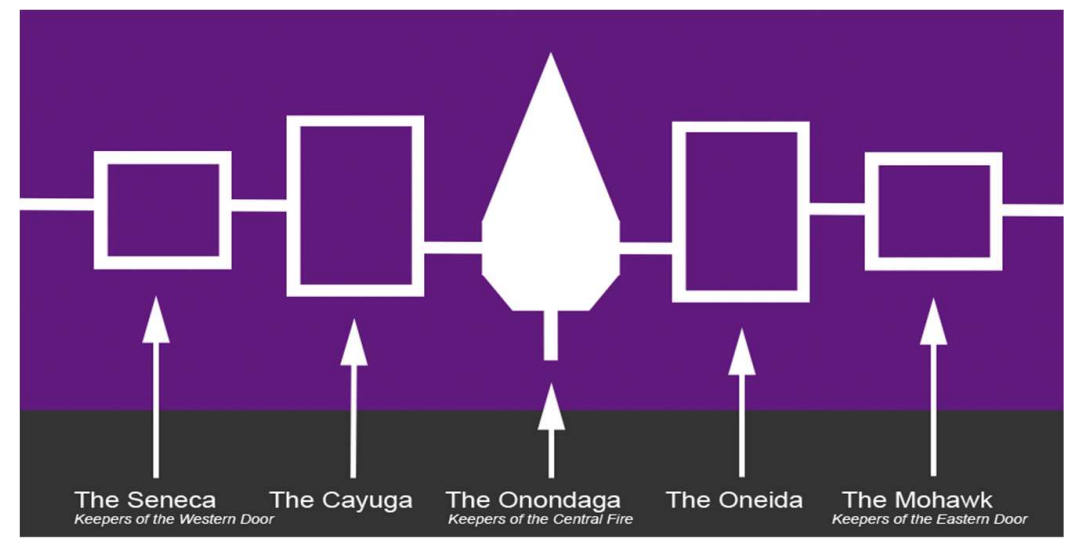

Bandeira da Confederação Haudenosaunee

Fonte: https://goo.gl/D7KVPW

De acordo com TEHANETORENS (1999, p. 11), a tradição oral conta que Hiawatha definiu como o 'wampum' seria usado, tendo ensinado às Cinco Nações originais que ele traria paz e uniria pela paz, substituindo o sangue. 0 'wampum' passa a ser considerado sagrado à medida que se torna mais comum e transformase em certificado de autoridade, garantindo a autenticidade de uma mensagem ou promessa.

A nação Onondaga, situada no centro do território Iroquois, foi escolhida para ser 'Keeper of the Sacred Fire', (Protetora do Fogo Sagrado), e também 'Keeper of the Wampum', (Protetora do Wampum). Como o 'wampum' faz parte da tradição oral, relacionando-se não só à palavra narrada, mas também à performance narrativa, duas vezes por ano, em um conselho, um Protetor reunia as pessoas, segurava os 'wampums' para que todos pudessem vê-los, e recitava a mensagem de cada um. O 'wampum' era recitado para que sua mensagem tivesse continuidade e para que jovens selecionados recebessem o treinamento adequado para conhecer e memorizar o significado do documento, tornando-se um dia também Protetores do Wampum. 
Entre os muitos 'wampum belts', destaca-se 'The Two Row Wampum Belt', ou 'Guswhenta/Kaswentha', de 1613, que fundamenta as relações entre índios e não índios e marca um acordo firmado entre as Cinco Nações Haudenosaunee originais e representantes do governo holandês estabelecido em Albany, localizada onde hoje fica o Estado de Nova Iorque.

'The Two Row Wampum Belt' consiste de duas colunas, com contas púrpura, em um fundo com contas de cor branca, simbolizando a paz. As contas de cor púrpura simbolizam o curso dos barcos dos europeus e das canoas indígenas, lado a lado, mas sem se tocar. 0 'wampum' representa a soberania de cada nação, bem como a igualdade em que se encontram. Os Haudenosaunee eram poderosos e a colônia era jovem, mas os nativos estavam se colocando como iguais e reconhecendo o outro povo como nação. Eram povos diferentes, cada qual com seu governo, suas leis e costumes. Uma interpretação das três colunas brancas é que significam amizade, paz e respeito para sempre entre as duas nações.

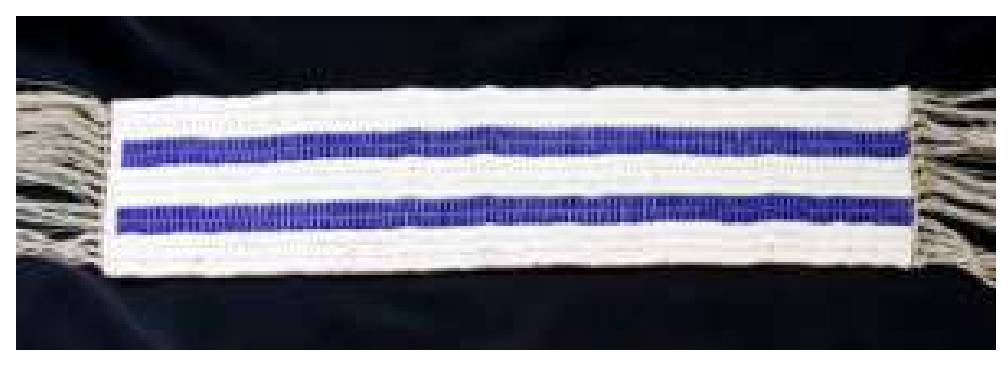

Two-Row Wampum Belt

Fonte: http://www.onondaganation.org/culture/wampum/two-row-wampum-belt-guswenta

Além de texto diplomático, o tratado construído pela tradição indígena, por sua multimodalidade, também poderia ser considerado texto literário produzido de acordo com convenções culturais nativas, representando o drama colonial pela forma e pelo conteúdo. Segundo Eric CHEYFITZ, “Em um livro publicado postumamente em 1942, Rourke afirma que os tratados anteriores à Guerra Revolucionária negociados entre colonos e índios 'são na verdade nossas primeiras 
peças de teatro americanas'[...], 'poesia colaborativa de alto nível' [...]."2 (2006, pp. 6-7, tradução nossa).

0 tratado constitui diálogo intercultural, mas também articula a temática da colonização pela maneira como encena as relações de poder entre nações estrangeiras. Os 'wampum belts' ainda hoje constituem símbolo relevante para as nações indígenas, pelo que significaram no passado, por fazerem parte da história, da memória indígena, além do que representam para o presente e para o futuro, para as próximas gerações.

A relação entre os povos indígenas e os povos colonizadores estabelecida por meio dos 'wampum belts' foi representada pela literatura indígena contemporânea por Eric Gansworth, que tece 'wampum belts' em poemas e imagens. A obra Nickel Eclipse: Iroquois moon pode ser lida como uma tradução intersemiótica da mitologia Haudenosaunee realizada por Gansworth. Sistemas de crenças que envolvem relações humanas e com o ambiente, questões comunitárias, éticas, morais e emocionais compõem a expressão estético-literária nativa interpretada pelos próprios nativos para suas comunidades e leitores de outras culturas. As mitologias são sagradas e contam histórias consideradas verdadeiras e vivas. Composições artísticas e literárias contemporâneas são geradas pelo conhecimento dessas mitologias e percepções ancestrais são transformadas pelas relações com o outro, relações de poder com nações colonizadoras e colonizadas, por questões sociais, políticas, econômicas e linguísticas.

Segundo Julio PLAZA (2003, p. 2), a tradução "se apresenta como 'a forma mais atenta de ler' a história porque é uma forma produtiva de consumo, ao mesmo tempo que relança para o futuro aqueles aspectos da história que realmente foram lidos e incorporados ao presente." Ao traduzir os 'wampum belts' utilizados nos tratados indígenas para a construção literária, Gansworth lê a história Haudenosaunee e atualiza a linguagem dos textos de partida ou textos originários, os 'wampum belts' e outros elementos da mitologia Haudenosaunee, criando um texto que inscreve a linguagem dos tratados poeticamente na

\footnotetext{
2 "In a book published posthumously in 1942, Rourke remarks that the pre-Revolutionary War treaties negotiated between colonists and Indians 'are in truth our first American plays' [...], a collaborative 'poetry of a high order' [...]."
} 
contemporaneidade. Além disso, PLAZA (2003, p. 8) afirma que, tratando da tradução, pode-se perceber "o passado como ícone, como possibilidade, como original a ser traduzido, o presente como índice, como tensão criativo-tradutora [...] e o futuro como símbolo, [...] a criação à procura de um leitor." Portanto, o artista/poeta indígena pode, pela literatura, traduzir os eventos históricos da colonização, lançar reflexões sobre a tensão tradutora de uma linguagem artística (e política) para outra, e formar leitores atentos à expressão multimodal da literatura indígena e às questões que permeiam esse discurso.

Por sua vez, a tradução intersemiótica é considerada por PLAZA (2003, p. 14) como "prática crítico-criativa na historicidade dos meios de produção e reprodução, como leitura, como metacriação, como ação sobre estruturas eventos, como diálogos de signos, como síntese e reescritura da história." Assim, ao traduzir a mitologia Haudenosaunee para a literatura contemporânea, Gansworth apresenta artisticamente não só uma transposição de um suporte ('wampum belt') para outro (livro com textos verbais e não-verbais), mas um diálogo entre signos que representam a continuidade da cultura indígena que, de forma crítica, como afirmação de resistência e sustentabilidade cultural, escrevem o apagamento (eclipse) e ressurgimento da cultura Haudenosaunee.

Eric Gansworth é um escritor e artista Onondaga que nasceu e cresceu em uma reserva Tuscarora em Lewiston, no estado de Nova Iorque, EUA. O autor começou sua carreira nas artes visuais, tendo alcançado grande sucesso. Entre suas obras de ficção destacam-se: Indian summers (1998), Smoke dancing (2004), Mending skins (2005), Extra Indians (2010) e If I ever get out of here (2013). Sua poesia inclui Nickel eclipse: Iroquois moon (2000) e A half-life of cardiopulmonar function (2008). Ele também escreveu peças de teatro que foram encenadas e uma obra com vários gêneros textuais intitulada Breathing the monster alive (2006). A visão estética de Gansworth está vinculada à iconografia e às tradições culturais Haudenosaunee. 0 autor se apropria de elementos da cultura Haudenosaunee, traduz essa cultura e retextualiza sua iconografia construindo resistência ao apagamento das culturas nativas e promovendo a autodeterminação. Na obra Nickel eclipse: Iroquois moon, Gansworth produz e integra duas formas artísticas, a poesia verbal e a pintura, o que permite ao leitor perceber diferentes dimensões da 
obra e as complexidades da composição indígena. Além disso, o autor/poeta/ilustrador reúne a narrativa histórica e a mítica, fazendo com que os mitos recebam novos sentidos no presente. A iconografia visual expressa por Gansworth pode ser observada na capa da obra Nickel eclipse: Iroquois moon, apresentada a seguir:

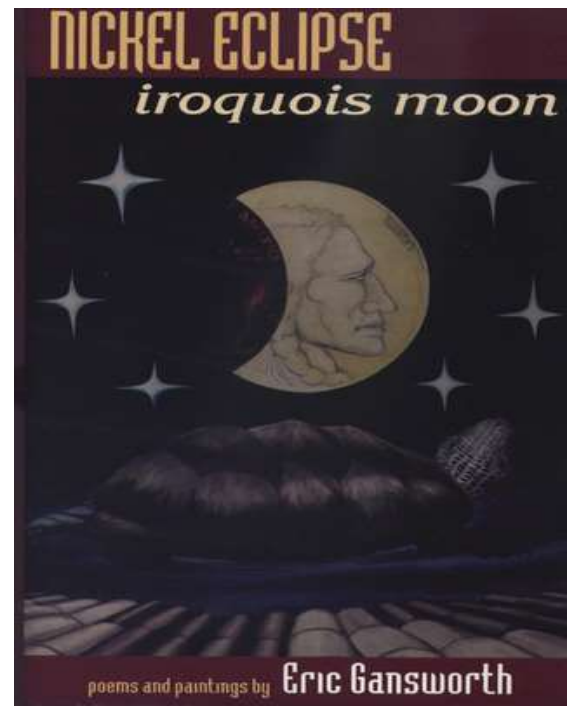

Capa da obra Nickel eclipse: Iroquois moon Fonte: https://goo.gl/62z11F

A imagem da capa do livro constrói uma tradução intersemiótica da iconografia Haudenosaunee pela presença de vários elementos: as contas de cor branca e púrpura do 'wampum', na parte de baixo da capa, a tartaruga que está vinculada ao calendário lunar Haudenosaunee e a moeda chamada Buffalo Nickel com a face do nativo americano.

0 título da obra propõe uma reflexão sobre o apagamento gradual das culturas nativas, mas aponta também para a observação da mitologia e da identidade Iroquois, que ganha visibilidade com uma face da moeda de 5 centavos, o 'Buffalo Nickel'. Essa moeda traz o perfil de um nativo em uma de suas faces, na cara, e uma imagem de um búfalo na coroa, sendo a única moeda denominada pela imagem da coroa. A imagem do búfalo, animal em vias de extinção em um dado momento, sobrepõe-se à imagem do nativo, apagado, eclipsado e quase extinto 
pela cultura dominante. Porém, o eclipse passa e a imagem apagada do nativo ressurge ao final da obra de Gansworth.

É relevante notar que, segundo Bruce Elliot JOHANSEN e Barbara Alice MANN (2000, p. 152) houve um eclipse de grande duração em 1142 que cobriu grande parte do território Haudenosaunee. Tal evento foi denominado pelos Haudenosaunee como 'Black Sun' (Sol Negro) e ocorreu antes da formação da Confederação. Portanto, um elemento cósmico pode ter sido lido pelos nativos da época como um sinal de que os povos precisariam unir-se.

A obra de Gansworth discutida nesse artigo é composta por uma coleção de poemas narrativos que refletem a tensão do eu poético que vive em uma cultura colonizadora, mas mantém a cultura ancestral. 0 livro é dividido em capítulos intitulados segundo o calendário das treze luas Haudenosaunee. 0 calendário observa uma série de cerimônias que coincidem com as estações do ano e são treze correspondendo ao desenho do casco da tartaruga, relevante na mitologia Haudenosaunee. Há muitas histórias de criação, provenientes da tradição oral, sobre a tartaruga, inclusive apontando como o animal carrega a terra nas costas. Segundo Jean CHEVALIER e Alain GHEERBRANT (1988, p. 868), "Pela sua carapaça, redonda como o céu na parte superior [...] e plana como a terra na parte inferior, a tartaruga é uma representação do universo: constitui-se por si mesma como uma cosmografia; [...]".

Pinturas aparecem no início de cada capítulo, relacionadas à história e à memória da nação Haudenosaunee, reforçando a presença do 'wampum' e da progressão do eclipse ao longo da construção poética. A relação dos grupos nativos com as culturas colonizadoras - da quase extinção ao ressurgimento - é simbolizada pelo eclipse da lua. A história Haudenosaunee é traduzida pelas imagens e versos, de seu início, crescimento e quase extinção, até seu ressurgimento, trazendo as vidas dos nativos não contemporâneos para o público contemporâneo, reconstruindo as lutas, a resistência e o reaparecimento da cultura e da vida nativa.

Além da representação de uma lua em diversas fases de um eclipse, do 'wampum belt' e da moeda com a face do nativo ou do búfalo, alguns símbolos relevantes para a cultura Haudenosaunee são utilizados nas imagens introdutórias 
dos capítulos, como três irmãos (milho, favas e abóbora), frutas vermelhas silvestres (morangos) e milho verde, relacionadas às cerimônias sagradas daqueles povos. Entretanto, apenas o 'wampum' e o eclipse são imagens constantes das pinturas.

A primeira imagem segue o poema de abertura, Nickel Eclipse, o qual dá o tom do texto, sugerindo o apagamento do indígena pela colonização e seu ressurgimento quando a imagem do búfalo fica na sombra e acontece a passagem do eclipse. Assim, a imagem inicial mostra 'The midwinter moon' (A lua da metade do inverno), na qual se vê a face de um índio em uma moeda-lua, na fase de lua cheia, com a inscrição 'Liberty' (liberdade) e com apenas pequena parte do 'wampum' podendo ser vista. Aparece ainda em destaque uma garrafa, a qual já pode sugerir uma representação do alcoolismo, presente na cultura indígena a partir da colonização.

Na segunda figura, vê-se o início do eclipse e a imagem do 'wampum' destaca-se um pouco. A terceira imagem mostra o nativo na moeda-lua coberto pelo eclipse e pela figura do búfalo, com o 'wampum' um pouco mais visível. Percebe-se, assim, um movimento da pintura, de presença da face indígena para seu apagamento pelo eclipse. Quanto mais a face do nativo é coberta, mais o 'wampum' se destaca. Portanto, a presença do indígena pode estar sendo apagada pela colonização, mas o 'wampum' mantém a identidade dos povos colonizados para que suas narrativas e história sobrevivam e ressurjam com a passagem do eclipse.

A imagem final traz novamente a representação da moeda-lua, em fase de lua cheia, com destaque para a face indígena, o que demonstra a resistência indígena diante das estratégias colonizadoras de apagamento de sua cultura, sua identidade e sua voz. O poema de Eric GANSWORTH que encerra a obra, It goes something like this (2000, p. 185), traz em seu último verso "Sometimes the story is enough to bring me home" ("Às vezes a história é suficiente para me levar para casa", tradução nossa), texto que pode ser interpretado como uma declaração de que são as histórias, narrativas, composições estético-literárias traduzidas para a contemporaneidade que sustentam a identidade e trazem o nativo para casa, para os laços da comunidade indígena. 


\section{Conclusão}

A obra Nickel eclipse: Iroquois moon de Gansworth constitui uma tessitura de elementos pictográficos que encenam o drama do eclipse e ressurgimento das nações e culturas nativas. 0 eclipse passa e as culturas e literaturas nativas ressurgem com sua representação estética na contemporaneidade. Portanto, a obra Nickel Eclipse: Iroquois moon traduz a resistência nativa diante da colonização, bem como a sustentabilidade cultural e a identidade Haudenosaunee expressa pela inserção de símbolos relevantes historicamente.

As literaturas das nações indígenas, em sua composição multicultural e multimodal, fazem com que leitores das mais diversas culturas redimensionem suas visões de mundo e, principalmente, suas perspectivas sobre as contribuições dos nativos para a construção de tradições estético-literárias das Américas.

\section{REFERÊNCIAS}

CHARLES River Editors. Native American tribes: the history and culture of the Iroquois confederacy. San Bernardino, California, USA: Charles River, 2017.

CHEVAliER, Jean GHEERBRANT, Alain. Dicionário de Símbolos: mitos, sonhos, costumes, gestos, formas, figuras, cores, números. Tradução de Vera da Costa e Silva [et al]. Rio de Janeiro: José Olympio, 1988.

CHEYFITZ, Eric. The Columbia guide to American Indian literatures of the United States since 1945. New York: Columbia University Press, 2006.

GANSWORTH, Eric. Nickel Eclipse: Iroquois moon: poems and illustrations. East Lansing, Michigan: Michigan State University Press, 2000.

HENSON, Eric C. [et al.] The state of the Native nations: conditions under U.S. policies of self-determination: the Harvard Project on American Indian Economic Development. New York: Oxford University Press, 2008.

JOHANSEN, Bruce Elliot; MANN, Barbara Alice (ed.). Encyclopedia of the Haudenosaunee (Iroquois Confederacy). Westport, CT, USA: Greenwood Press, 2000. 
MOMADAY, N. Scott. The man made of words: essays, stories, passages. New York: St. Martin's Press, 1997.

PERKINS, George et al. (ed). Harper Collins Reader's encyclopaedia of American literature. 2 ed. New York: Harper Collins, 2002.

PLAZA, Julio. Tradução intersemiótica. Coleção Estudos, n. 93. São Paulo: Perspectiva, 2003.

RISÉRIO, Antonio. Textos e tribos: poéticas extraocidentais nos trópicos brasileiros. Série Diversos. Rio de Janeiro: Imago, 1993.

TEHANETORENS (RAY FADDEN). Wampum belts of the Iroquois. Summertown, TN: Book Publishing Company, 1999.

\section{Sites consultados:}

BANDEIRA da Confederação Haudenosaune. Disponível em: <http://www.haudenosauneeconfederacy.com/confederacycreation.html > Acesso em: 10 set. 2017.

NICKEL ECLIPSE: IROQUOIS MOON. Capa do livro (2000). Disponível em: <https://books.google.com.br/books/about/Nickel Eclipse.html?id=idhaAAAAMA AJ\&redir esc $=y>$ Acesso em: 25 abr. 2018.

TWO-ROW Wampum Belt. Disponível <http://www.onondaganation.org/culture/wampum/two-row-wampum-beltguswenta > Acesso em: 10 set. 2017.

\section{Biografia da autora}

Janice Cristine Thiél possui graduação em Letras Português Inglês pela Universidade Federal do Paraná (1984), Mestrado em Letras pela Universidade Federal do Paraná (1989) e doutorado em Letras pela Universidade Federal do Paraná (2006). Atualmente é professora titular, da área de Letras, da Pontifícia Universidade Católica do Paraná e realiza Pós-Doutorado na UFPR. Atuou como professora do Mestrado em Teoria Literária do Centro Universitário Campos de Andrade de 2010 a 2012. Tem experiência na área de Letras, com ênfase em Literaturas Estrangeiras Modernas, atuando principalmente nos seguintes temas: alteridade, literatura indígena, literaturas de língua inglesa, leitura, multiletramento tradução e estudos culturais.

Recebido em: 29/03/2018

Aceito em: 28/04/2018

Publicado em junho de 2018 